\title{
A Soft Computing based Adaptive Call Admission Control Scheme for Bi-class Traffic in CDMA Cellular Network
}

\author{
Ravi Sindal \\ Institute of Engineering and Technology \\ Devi Ahilya University \\ Khandwa Road, Indore, M.P
}

\author{
Sanjiv Tokekar \\ Institute of Engineering and Technology \\ Devi Ahilya University \\ Khandwa Road, Indore, M.P
}

\begin{abstract}
Soft handoff in CDMA cellular system increases system capacity as compared to hard handoff as interference is reduced by transmitting signals as lower power level. A CDMA cellular system involving voice and data calls is considered in this paper. The model proposed in this paper is for data and voice services (Bi-class). It is based on reservation for handoff voice and data calls along with call queuing scheme for handoff data calls. Preemptive priority scheme is used for handoff voice calls over ongoing data calls. Upper channel limits are set for new voice as well as data calls to give priority to handoff calls. For better resource management and efficient call admission to originating and handoff calls, Neuro-fuzzy call admission controller is designed. This controller uses the adaptable feature of soft handoff threshold parameters to accommodate important voice and data calls. The proposed ASNFC controller is compared with the two variants of Bi-class CAC scheme.
\end{abstract}

\section{General Terms}

Call Admission Control, Soft Computing

\section{Keywords}

Soft Handoff, CDMA, Neuro-Fuzzy, Adaptive.

\section{INTRODUCTION}

The emergences of $3 \mathrm{G}$ technologies and development in advance mobile communication technologies have enabled the users to use multi-service features on same network. CDMA (Code division multiple access), which is a multiple access technique has emerged as one of the better techniques in providing these services due to flexibility in transmission rates and better handover features. Handoff is the process of transferring a MS (Mobile station) from one BS (Base station) or channel to other. When a mobile station during active call moves from one cell to another, radio resources need to be transferred to new base station. Macro diversity is provided in CDMA cellular network by this characteristic of soft handoff [1]. In previous papers, we have modeled two dimensional markov model for Bi-class services mainly voice and data services (new as well as handoff calls) for CDMA cellular network .These models uses partial channel reservation policies for both type of services. These models were analyzed for variation in reserved channels for voice calls and soft handoff threshold parameters. The main aim of this paper is to give a comparative analysis of proposed Neuro-fuzzy CAC with previously proposed analytical models for Bi-class services sharing the common channels in the network. The two types of services considered here are voice and data calls.
Real time services like voice calls can not tolerate long delays and need continuous connection. Whereas non real time calls like data calls can tolerate delays and can be buffered till resources are available [3].

\section{SOFT HANDOFF THRESHOLDS}

The main purpose of a soft handover scheme is to assure that there is connectivity with the previous BS while the new BS has been assigned to take control over the communication link during the travel of MS. For initiating the soft handoff mechanism in CDMA cellular network, pilot signal strength in form of chip-energy-to-interference ratio (EC/Io) is measured by MS for each BS. These received pilots from different MS are stored in four different sets mainly: active, neighbor, candidate and remaining [2].

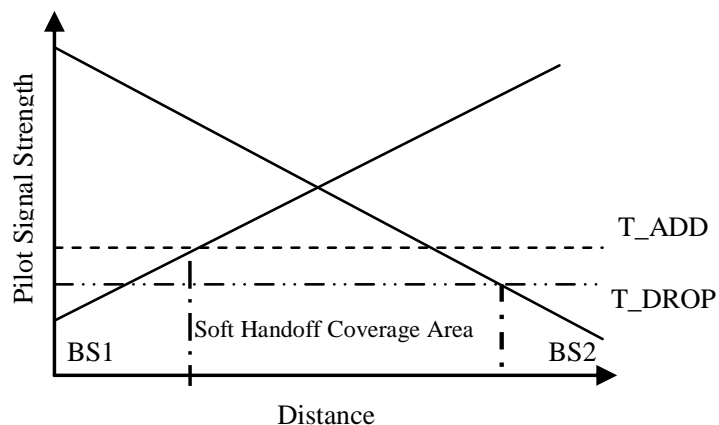

Fig 1: Soft Handoff region in CDMA Cellular Network

Threshold parameters settings named as T_ADD and T_DROP are set depending on soft handoff coverage area and BS transmitting power to shift received pilots in any of the four sets stated above. A pilot in the neighbor or remaining set is moved to the candidate set, if its $\mathrm{E}_{\mathrm{C}} / \mathrm{I}_{\mathrm{o}}$ is greater than T_ADD. A pilot in the active or candidate set is moved to the neighbor set, if its $E_{C} / I_{0}$ falls below T_DROP for a period of $\mathrm{T}$ TDROP seconds [2]. These two levels of threshold are chosen to avoid abrupt call transfer during the travel. Due to fact that adjacent cells in cellular system are overlaid, handoff queuing scheme came into picture. The Fig.1 above shows the variation in soft handoff coverage area for change in threshold parameters. As MS moves away from parent BS, the received signal strength decreases and when it gets worsen than threshold value T_DROP, the handoff procedure is initiated and MS is handed over to next BS. When received signal strength is between T_ADD and T_DROP, the MS is connected to multiple BS. During the period the MS is in soft handoff coverage area, it is connected to both the BS. This 
situation is similar to a call entering in a queue and waiting for a free channel. The capacity of queue for handling data handoff calls can be varied by varying the two threshold parameters [1] [2].

\section{STOCHASTIC MODEL}

We have considered an analytical model of a single cell in a cellular wireless communication network [3]. For the CDMA cellular network, let ' $\mathrm{C}$ ' be the limited amount of code channels available in the channel pool. Full channel availability is made for both voice handoff calls and data handoff calls. The number of channels made available for new voice call and new data call is limited by two upper channel limits for each set of calls. The upper channel limit for new voice call is given by 'Uvn' and for new data call it is 'Udn'. Whenever a new voice or data call requests for channel and if number of channels in use for these services currently is equal to the upper limit allocated to them, then the requesting new call is blocked. For handling the data handoff calls, queuing scheme is used. A data handoff request is put in the queue if BS finds that all channels in target cell are occupied. If a channel is released when the queue for data handoff requests is not empty, the channel is assigned to request on the top of the queue. The queue for data handoff call can be realized due to nature of soft handoff coverage area.

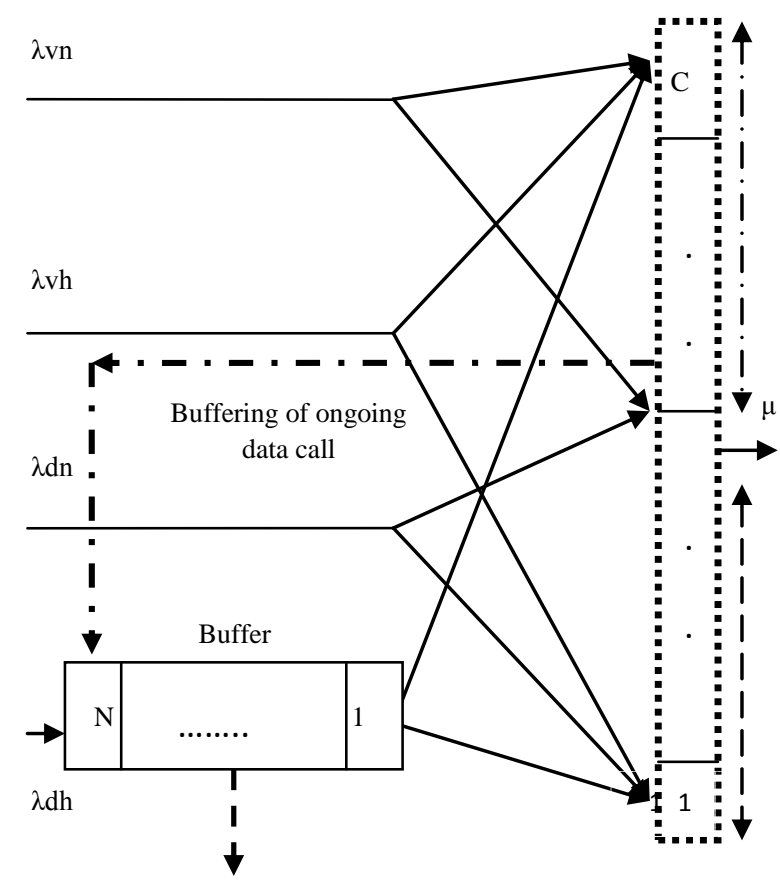

Forced Termination

Code Channels

Fig 2: Bi-class Model (Preemptive scheme)

Queuing scheme is considered for data handoff calls as they are non-real time calls and delay can be tolerated for these types of services. The queue capacity is decided by the setting of the two soft handoff threshold parameters mentioned previously. Larger is the difference in these parameters, greater is the queue capacity. A finite queue with capacity ' $\mathrm{N}$ ' and FIFO (First in First out) characteristic is assumed at the Base Stations. The System model with limits for new calls and finite queue for data handoff call is shown in Fig.2 [4] [5].

\subsection{Traffic Model}

For the analytical model, following assumptions are taken into consideration.

i) The total arrival rate of voice traffic is given by $\lambda v=\lambda v n+$ $\lambda$ vh.

ii) The total arrival rate of data traffic is given by $\lambda d=\lambda d n+$ $\lambda \mathrm{dh}$.

iii) The channel holding time is considered to be exponentially distributed with mean rate ' $\mu$ '.

iv) For new voice and data calls upper limits for channel utilization is set by setting value of Uvn and Udn.

v) The sum of upper limits for new voice and data call is less than total number of code channels available. ie

$$
\text { Unv+Und }<<\mathrm{C}
$$

vi) Finite buffer capacity is assumed for data handoff calls and it depends on soft handoff threshold parameters.

vii) Preemptive scheme is used for handoff voice call.

\subsection{Model Analysis}

We can define the state of system by $(\mathrm{Cv}, \mathrm{Cd})$, where $\mathrm{Cv}$ is the total number of voice calls (both new and handoff calls) and $\mathrm{Cd}$ is the number of data calls ( being served as well as handoff data call waiting in queue). Through this assumption, $(\mathrm{Cv}, \mathrm{Cd})$ is a two dimensional Markov chain[3].The state transition diagram for the model is shown in Fig.3.The total number of voice call (both new and handoff) in any state varies form 0 to C. Similarly total number of data calls (new and handoff) varies from 0 to $\mathrm{C}+\mathrm{N}$. Since it is finite two dimensional birth-death model, sum of all state probabilities $\mathrm{P}_{(\mathrm{Cv}, \mathrm{Cd})}$ is equal to ' 1 ' [4].

For determining the steady state probabilities, the generalized equation for Multi-class model which is continuous time homogenous Markov chain can be accordingly modified [5]. By using the relations for the steady state probabilities $\mathrm{P}(\mathrm{Cv}, \mathrm{Cd})$, the performance parameters can be calculated for the proposed model. The blocking probability of new voice call can be calculated by determining the condition when the number of channels occupied by new voice call has reached the set upper limit $U_{v n}$

$$
P(\text { block })_{v n}=\left[\sum_{C v=U v n}^{C} \sum_{C d=0}^{C+N-C v} P_{(C v, C d)}\right] * R_{v n}
$$

The blocking probability of new data call is that probability when the busy channels for ongoing data calls equal the upper set limit $\mathrm{U}_{\mathrm{dn}}$. 

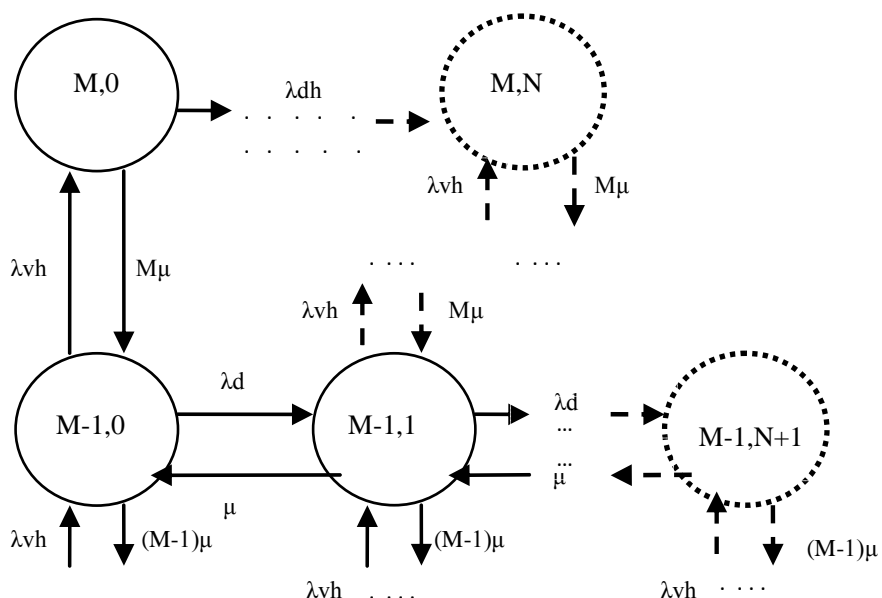

Channel in use

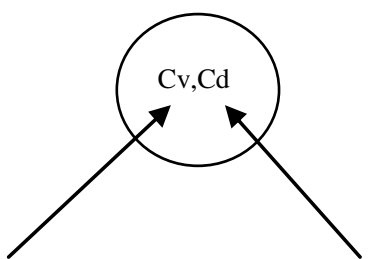

for voice calls

Channel in use

for data calls
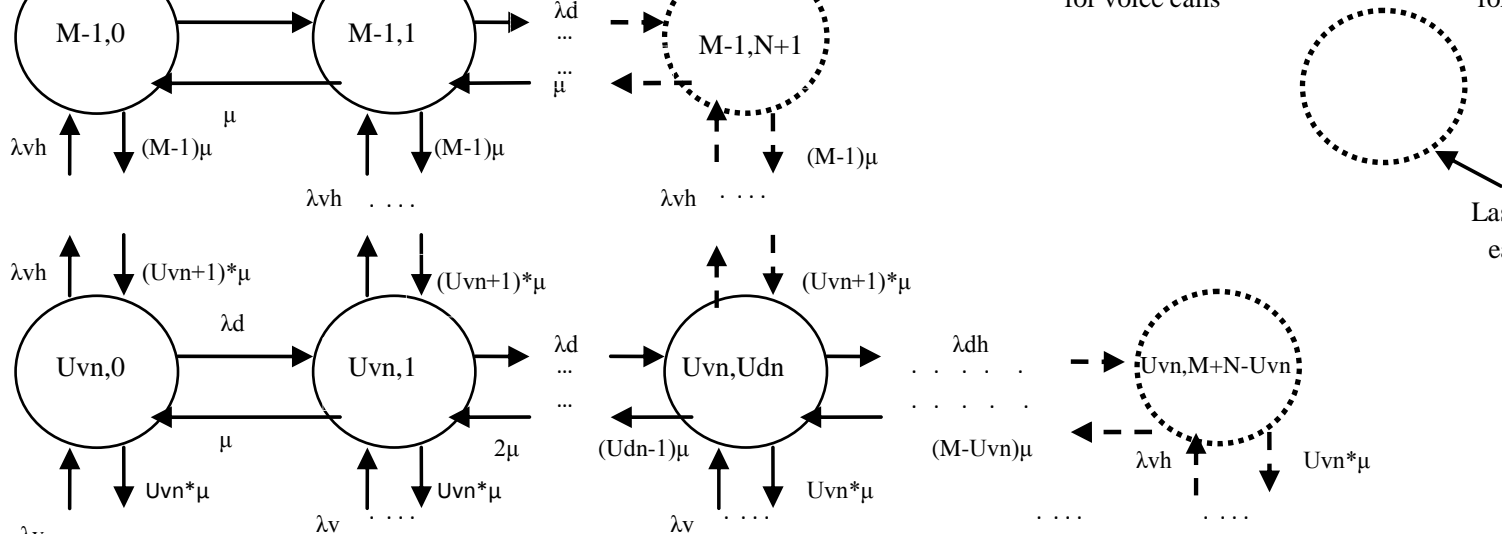

Last state in each row
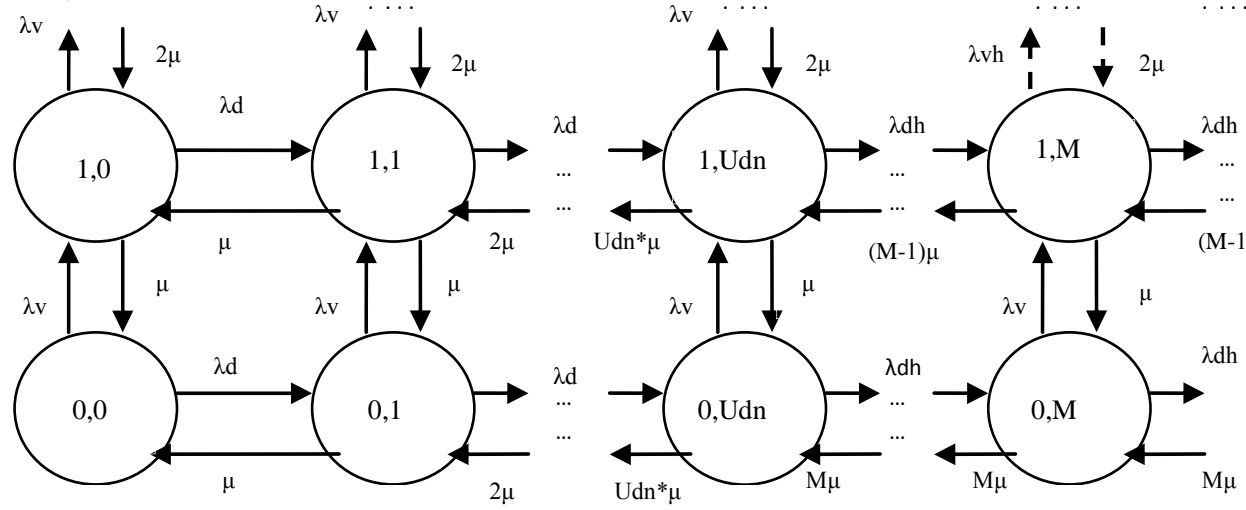

Fig 3: Two dimensional Markov chain for Bi-class Model

$P(\text { block })_{d n}=\left[\sum_{C v=0}^{C} \sum_{C d=U d n}^{C+N-C v} P_{(C v, C d)}\right] * R_{d n}$

Handoff Voice call dropping probability is given by the situation when all the ' $\mathrm{C}$ ' channels are busy and no channel is available to serve the incoming handoff voice request.

$P(\text { drop })_{v h}=\left[\sum_{C v=0}^{C} P_{(C v, C+N-C v)}\right] * R_{v h}$
Similarly, the handoff data call dropping probability can be evaluated by the situation when all channels are busy and no slot is available in the data handoff queue.

$$
P(\text { drop })_{d h}=\left[\sum_{C v=0}^{C} P_{(C v, C+N-C v)}\right] * R_{d h}
$$

Where $R_{v n}, R_{d n}, R_{v h}$ and $R_{d h}$ represents relative mobility for new voice, new data, handoff voice and handoff data calls[5]. 


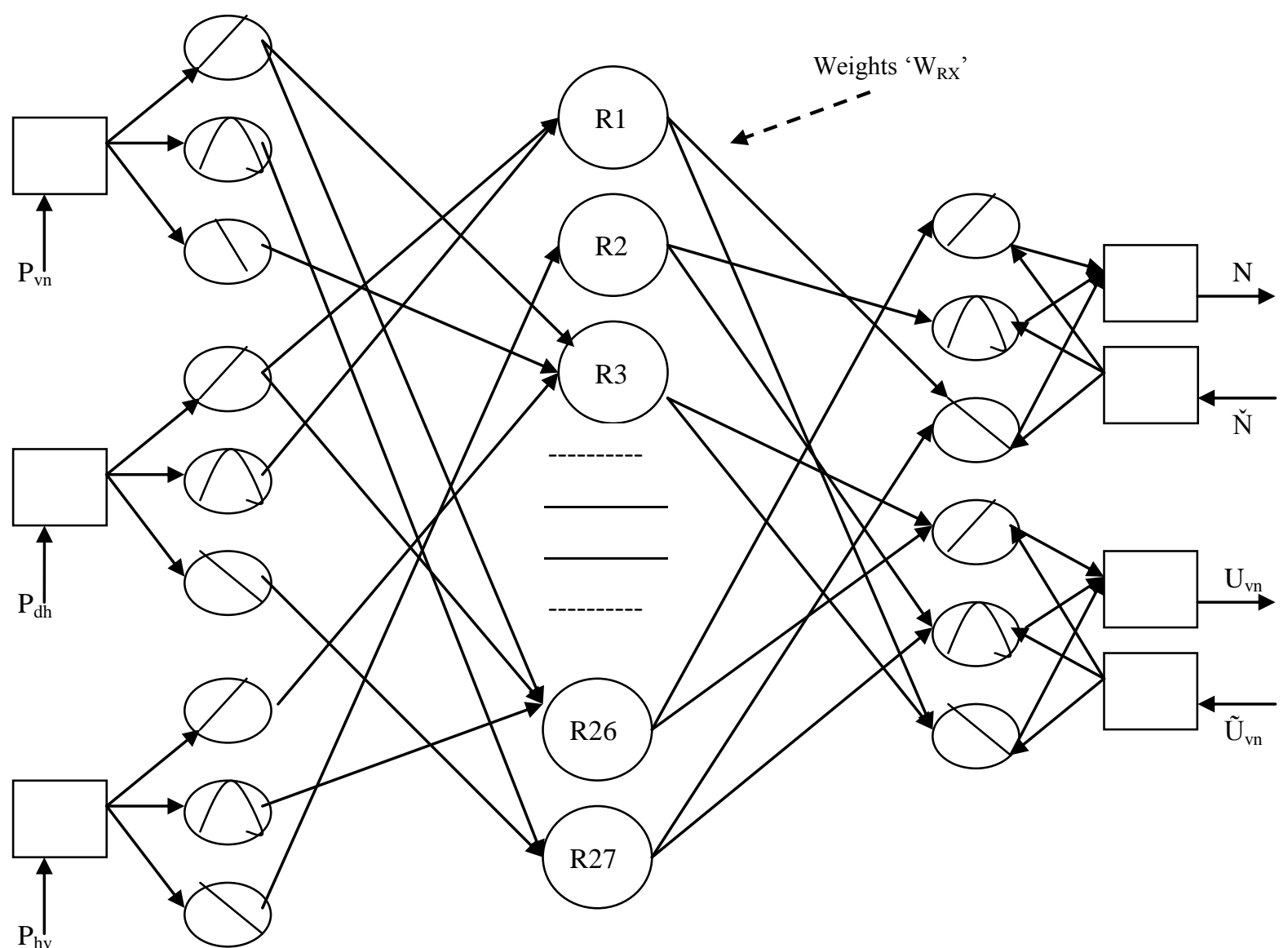

Fig 4: Structure of ASNFC Controller

\section{ASNFC DESIGN}

The Neuro-fuzzy model developed uses the training data from the analytical model and termed as Adaptive Soft handoff based Neuro-Fuzzy Call Admission Controller (ASNFC). ASNFC uses new voice blocking probability $\left(\mathrm{P}_{\mathrm{vn}}\right)$, handoff data dropping probability $\left(\mathrm{P}_{\mathrm{hd}}\right)$ and handoff voice call traffic $\left(\mathrm{T}_{\mathrm{hv}}\right)$ as input linguistic variables. The output linguistic variables are handoff data call queue capacity $(\mathrm{N})$ and new voice call threshold $\left(\mathrm{U}_{\mathrm{vn}}\right)$. Five layer neuro-fuzzy controller architecture is used to design the ASNFC [6]. The structure of a neuro-fuzzy system is similar to a multi-layer neural network. In general, a neuro-fuzzy system has input and output layers, and three hidden layers that represent membership functions and fuzzy rules. Each layer in the ASNFC system is associated with a particular step in fuzzy inference process. Trapezoidal as well as triangular membership functions are used to define input-output linguistic variables. The terms used to define input linguistic variables are; $\mathrm{P}_{\mathrm{vn}}(0.0001$ to 1.0$)=\{$ Low $(\mathrm{L})$, Medium $(\mathrm{M})$, High $(\mathrm{H})\}, \mathrm{P}_{\text {hd }}(0.0001$ to 1.0$)=\{$ Low $(\mathrm{L})$, Medium $(\mathrm{M})$, High $(\mathrm{H})\}$, and $\mathrm{T}_{\text {hv }}(0$ to 100 erlang $)=\{$ Slow $(\mathrm{S})$, Moderate $(\mathrm{M})$, Fast $(\mathrm{F})\}$. The output linguistic variables used are: handoff data call queue capacity ' $\mathrm{N}$ ' (0 to 20$)=\{$ Low (L), Medium (M), High (H)\}, and new voice call threshold 'Uvn' (0 to 10) $=\{$ Low $(\mathrm{L})$, Medium $(\mathrm{M})$, High $(\mathrm{H})\}$. Triangular membership function for center variables and trapezoidal membership function for variables on extremities are chosen as analysis using these types of functions is practical and easy.The details of five layers as shown in Fig.4.
The rule base (total possible rules $=3^{3}=27$ ) for ASNFC is shown in table no.1.

Each neuron in the defuzzification layer represents a single output of the neuro-fuzzy system. It takes the output fuzzy sets clipped by the respective integrated firing strengths and combines them into a single fuzzy set. This neuro-fuzzy system applies standard defuzzification methods, the CoG technique.

Table 1: Rule base for ASNFC

\begin{tabular}{|c|c|c|c|c|c|}
\hline \multirow{2}{*}{ RULE } & \multicolumn{3}{|c|}{ IF } & \multicolumn{2}{c|}{ THEN } \\
\cline { 2 - 6 } & $\mathbf{P}_{\text {dh }}$ & $\mathbf{P}_{\mathbf{v n}}$ & $\mathbf{T}_{\text {hv }}$ & $\mathbf{N}$ & $\mathbf{U}_{\mathbf{v n}}$ \\
\hline R1 & High & High & Slow & High & High \\
\hline R2 & High & High & Moderate & Medium & High \\
\hline R3 & High & High & Fast & Medium & High \\
\hline R4 & High & Medium & Slow & High & Medium \\
\hline R5 & High & Medium & Moderate & High & Medium \\
\hline R6 & High & Medium & Fast & High & Medium \\
\hline R7 & High & Low & Slow & High & Low \\
\hline R8 & High & Low & Moderate & High & Low \\
\hline R9 & High & Low & Fast & High & Medium \\
\hline R10 & Medium & High & Slow & Medium & High \\
\hline R11 & Medium & High & Moderate & Medium & Medium \\
\hline
\end{tabular}




\begin{tabular}{|c|c|c|c|c|c|}
\hline R12 & Medium & High & Fast & Medium & High \\
\hline R13 & Medium & Medium & Slow & High & Medium \\
\hline R14 & Medium & Medium & Moderate & Medium & Low \\
\hline R15 & Medium & Medium & Fast & Medium & Low \\
\hline R16 & Medium & Low & Slow & Medium & Medium \\
\hline R17 & Medium & Low & Moderate & Medium & Medium \\
\hline R18 & Medium & Low & Fast & Medium & Low \\
\hline R19 & Low & High & Slow & Low & High \\
\hline R20 & Low & High & Moderate & Low & High \\
\hline R21 & Low & High & Fast & Low & High \\
\hline R22 & Low & Medium & Slow & Low & Medium \\
\hline R23 & Low & Medium & Moderate & Low & Medium \\
\hline R24 & Low & Medium & Fast & Low & Low \\
\hline R25 & Low & Low & Slow & Low & Low \\
\hline R26 & Low & Low & Moderate & Low & Medium \\
\hline R27 & Low & Low & Fast & Low & Low \\
\hline
\end{tabular}

\subsection{Training of ASNFC}

A total of 27 rules have been developed depending on different combinations of input-output linguistic parameters. As all the rules might not be realistic in a practical scenario, so the weights associated with individual rules have to be modified.

When input and output linguistic values are applied during training of the Neuro-fuzzy model, the system automatically generates a complete set of fuzzy IF-THEN rules. As expert knowledge is not embodied in the system this time, the initial weights between Layer 3 and Layer 4 are all set to value 1.0. After training those rules are eliminated whose certainty factors are less than a sufficiently small number, i.e 0.1 .The weights are modified through learning or training process. For training of the model, backpropogation algorithm has been used. The training continues until the sum of squared errors is less than 0.001[7].

For the training of the proposed neuro-fuzzy controller the training data set obtained from the analytical model mentioned in section 3 is used:

Table 2: Rule base for ASNFC model after training

\begin{tabular}{|c|c|c|c|c|c|c|}
\hline \multirow{2}{*}{ R. } & \multicolumn{3}{|c|}{ IF } & \multicolumn{2}{c|}{ THEN } & Wt \\
\cline { 2 - 6 } No & \multirow{2}{*}{$\mathbf{P}_{\text {dh }}$} & \multirow{2}{*}{$\mathbf{P}_{\text {vn }}$} & $\mathbf{T}_{\text {hv }}$ & $\mathbf{N}$ & $\mathbf{U}_{\mathbf{v n}}$ & Trainin \\
& & & & & & $\mathbf{g}$ \\
& & & & & & \\
\hline R4 & High & Mediu & Slow & High & Mediu & 0.54 \\
\hline R6 & High & Mediu & Fast & High & Mediu & 0.73 \\
\hline R1 & High & Low & Modera & High & Low & 0.92 \\
\hline R1 & Mediu & Mediu & Slow & High & Mediu & 0.68 \\
\hline R1 & Low & High & Slow & Low & High & 0.83 \\
\hline R2 & Low & High & Modera & Low & High & 0.35 \\
\hline R2 & Low & Mediu & Fast & Low & Low & 0.53 \\
\hline
\end{tabular}

\section{RESULTS AND DISCUSSION}

The proposed Neuro-fuzzy CAC model is compared with two variants of Bi-class CAC Analytical model. One model considers Preemptive policy for handoff voice calls whereas other considers non-preemptive policy [4]. The simulation and analytical results are obtained using MATLAB. All the models are analyzed for the input data ie $\mathrm{C}=64, \mathrm{U}_{\mathrm{vn}}=10$, $\mathrm{U}_{\mathrm{dn}}=10$ and $\mathrm{N}=6$.

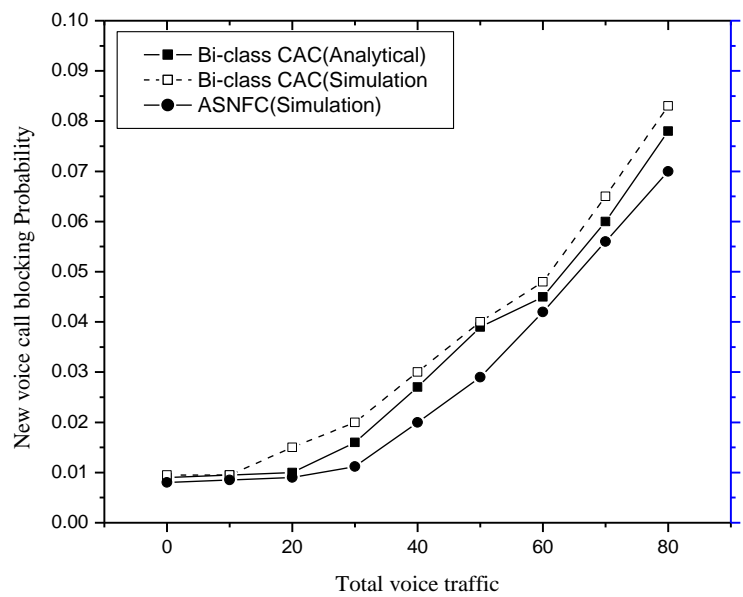

Fig: 5 New voice blocking probability v/s total voice traffic

The variation in handoff voice dropping probability for change in voice traffic is shown in Fig 6. The results obtained for Bi-class CAC (Preemptive) model are superior as compared to other two models. This is due to the fact that in preemptive scheme, the channel occupied by ongoing handoff call is released for handoff voice call when all channels are occupied.

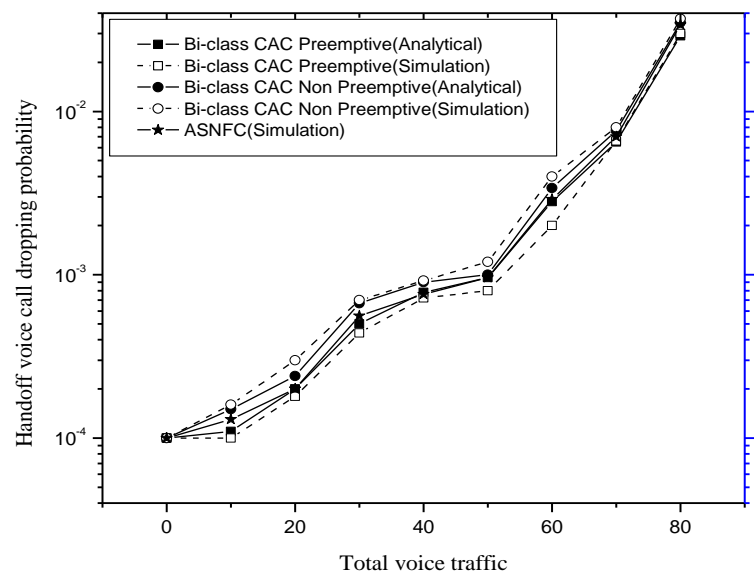

Fig 6: Handoff voice dropping probability v/s total voice traffic

Fig 7 shows the variation in new data call blocking for variation in total data traffic. The data call blocking probabilities offered by ASNFC model are lower as compared to the stochastic model. This is due to the fact that the neurofuzzy model dynamically adjusts the upper channel limit $\left(\mathrm{U}_{\mathrm{dn}}\right)$ reserved for the new data calls in case of arrival of heavy data 
traffic. There is a decrement in data call dropping probability by $25 \%$ for a traffic load of 80 erlang.

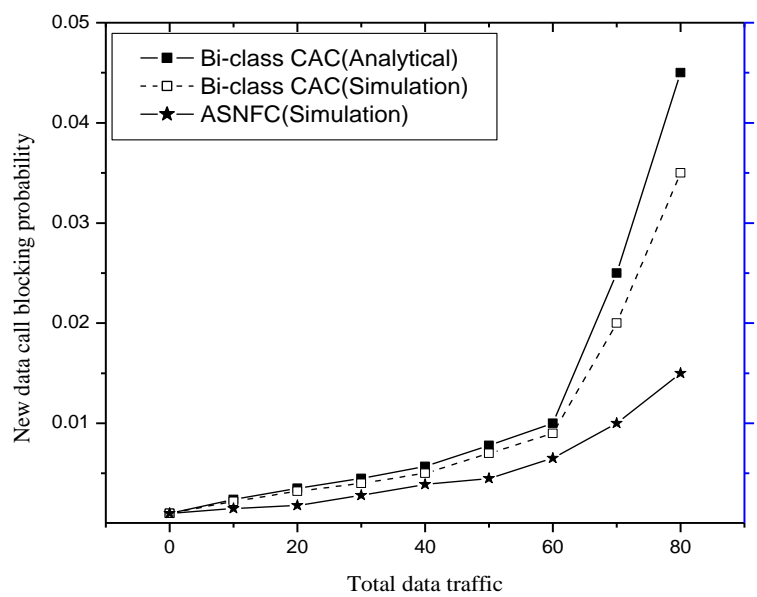

Fig 7: New data call blocking probability v/s total data traffic

Fig 8 gives a comparative analysis of handoff data dropping probability w.r.t incoming data traffic for the two models. The ASNFC model has the ability of modifying the soft handoff coverage area by changing the soft handoff threshold parameters. Due to this fact, it can dynamically increase the handoff data queue capacity to provide services to more calls. There is an improvement in handoff data dropping probability by $55 \%$. The variation in handoff data call dropping probability for change in voice traffic is illustrated in Fig 9.

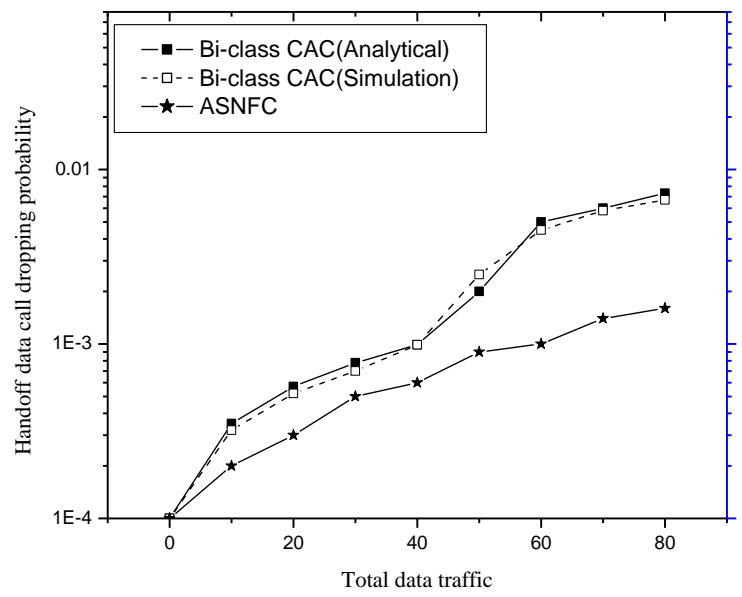

Fig 8: Handoff data call dropping probability v/s data traffic

The result shows that handoff data call dropping probability is immune to arrival voice traffic in case of ASNFC model. It increases in case of Bi-class (Preemptive) model as channel to incoming handoff voice traffic is made available in by releasing channel from an ongoing data call and putting it in queue.

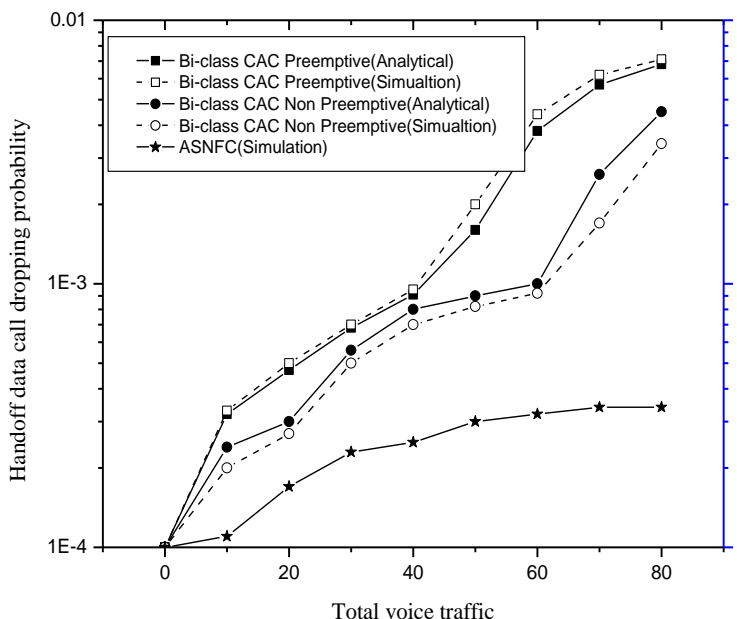

Fig:9 Handoff data call dropping probability v/s voice traffic

Fig 10 shows the effect of incoming traffic on forced termination probability of data calls. The Bi-class CAC models (preemptive and non-preemptive) have more forced termination of data calls as the handoff queue size can not be dynamically varied. The probability of forced termination is largest in case of preemptive model as ongoing data call has to release channel for incoming handoff voice call. The result is that the waiting period for accessing free channel as well as probability of forced termination increases. The percentage channel utilization by the two schemes is visualized in fig 11 . As the neuro-fuzzy algorithm makes dynamical fuzzy decision of the threshold values, so it provides better cell utilization for same values of incoming traffic.

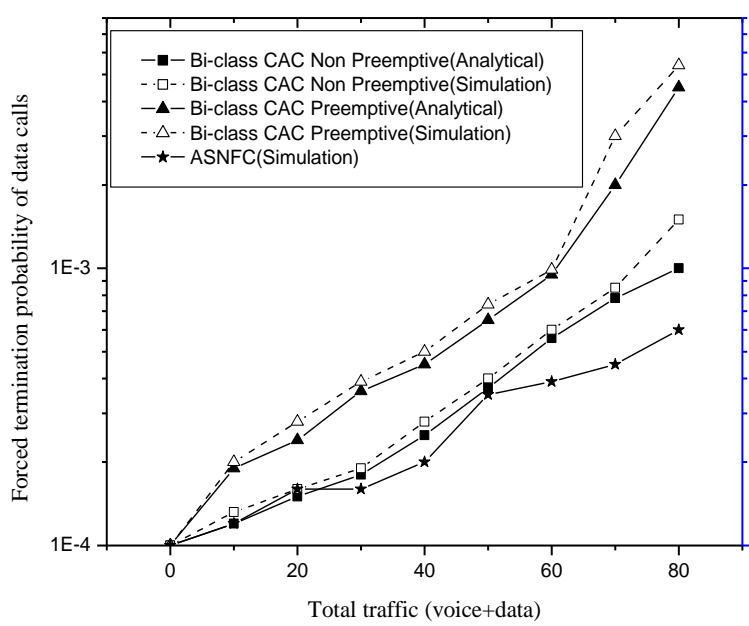

Fig :10 Forced termination probability of data call v/s total traffic 


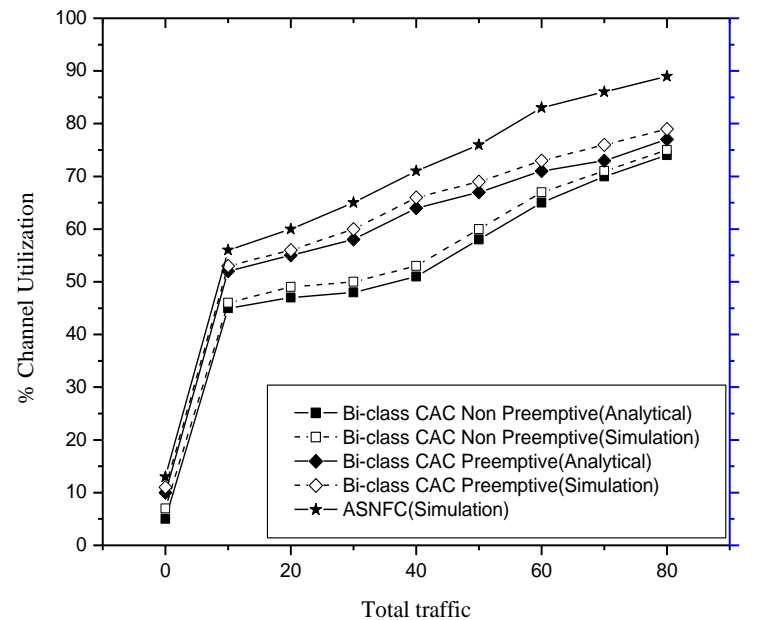

Fig:11 Percentage channel utilization v/s total traffic

\section{CONCLUSION}

The Bi-class stochastic models for call admission control of voice and data calls in CDMA cellular network are developed and analyzed for variation in handoff call queue size, incoming traffic and channel reserved for new voice and data calls. Two models are considered which uses non-preemptive and preemptive scheme for handling handoff voice calls. These models are also compared with Adaptive Soft handoff based Neuro-Fuzzy Call Admission Controller (ASNFC). The training of ASNFC model is done using dataset obtained from the Bi-class stochastic model. On analysis and simulation ,the results showed that the neuro-fuzzy model due to its adaptable nature of adjusting the soft handoff coverage region offer better results for the performance measure parameters. The performance parameters used for comparative analysis and simulation were new voice and data call blocking probability, handoff voice and data call dropping probability, forced termination probability of handoff data call and percentage channel utilization.

\section{REFERENCES}

[1] Jens Zander, Seong- Lyun Kim: Radio Resource Management for Wireless Networks,Aretch House Publishers Boston (2001).

[2] B.Worley, F.Takawira: Handoff schemes in CDMA cellular systems, IEEE Transections, (1998) 255-260.

[3] Ivan Stojmenovic: Handbook of Wireless Networks and Mobile Computing, John Wiley and Sons Inc (2002).

[4] Vrinda Tokekar, A.K Ramani and Sanjiv Tokekar,: Analysis of Batching Policy in View of User Reneging in VOD System, IEEE Indicon Conference, India(2005) 399-403.

[5] Ravi Sindal and Sanjiv Tokekar : Modeling and analysis of voice/data call admission control scheme in CDMA cellular network for variation in soft handoff threshold parameters, $16^{\text {th }}$ IEEE International Conference on Networks, (ICON 2008), New Delhi(2008).

[6] Kuen-Rong Lo, Chung-Ju Chang and C.Bernard Shung: A neural fuzzy resource manager for hierarchical cellular systems supporting multimedia services, IEEE Trans. on Veh. Tech., vol.52, no.5, (2003) 1196-1206.

[7] Chng-Ju Chang, Li- Chung Kuo, Yih-Shen Chen and Scott Shen: Neural fuzzy call admission and rate controller for WCDMA cellular systems providing multirate services,IWCMC'06 (2006) 383-388. 